\title{
促癌因子TRIM59不依赖泛素化酶活性促进乳腺癌 转移的机制
}

\author{
蒋德伟，陈策实 ${ }^{*}$ \\ 中国科学院动物模型与人类疾病机理重点实验室, 中国科学院昆明动物研究所, 昆明 650223 \\ *联系人, Email: chenc@mail.kiz.ac.cn
}

收稿日期：2018-12-10; 接受日期：2018-12-23; 网络版发表日期：2019-01-17

据2018全球癌症年报统计, 乳腺癌是女性中发病 率和死亡率最高的癌症 ${ }^{[1]}$. 在癌症进程中, 肿瘤细胞会 采取多种策略应对并适应微环境压力最终发生转移, 而肿瘤细胞转移是癌症病人死亡的主要原因 ${ }^{[2]}$. 临床 上发生癌细胞淋巴结和骨转移的乳腺癌患者预后生存 情况均较差. 泛素化系统对于维持细胞稳态和正常生 理功能十分重要. 很多重要功能蛋白的泛素化调控异 常已被证实是癌症发生的主要驱动因素之一 ${ }^{[3]}$. 在乳 腺癌发生发展过程中，关于泛素化介导的蛋白酶体降 解和自噬降解途径的机制研究已被广泛报道，但对于 肿瘤细胞迁移相关的泛素化调控研究还较为有限 ${ }^{[4,5]}$.

TRIM蛋白家族(The tripartite motif protein family) 是一类与肿瘤发生相关的E3泛素连接酶 ${ }^{[6]}$, 其在调节 肿瘤细胞的细胞周期及代谢稳态中发挥重要作用, 但 在肿瘤细胞侵袭和转移方面的研究却未见报道. 最近, 浙江大学韩卫东教授、德克萨斯农工大学Yubin Zhou 教授和德州大学健康中心Leng Han教授团队合作发现 了 TRIM59蛋白通过影响自噬介导的蛋白降解从而促 进乳腺癌转移的新机制 ${ }^{[7]}$.

通过TCGA(the Cancer Genome Atlas)数据库分析, 聚焦于TRIM家族近70个成员，研究者发现TRIM59在 12 种癌组织特别是乳腺癌中高表达. 分析 87 例临床样
本后发现, TRIM59的异常高表达与乳腺癌转移和恶 性程度呈正相关，与病人预后生存时间呈负相关. 在 乳腺癌细胞MCF-7中, 敲降或敲除TRIM59可抑制细胞 增殖、迁移并增加细胞凋亡, 同时也显著减弱其在裸 鼠上的成瘤和转移能力. 然而在MDA-MB-231细胞中, 过表达TRIM59则观察到相反的结果. 研究者还发现 TRIM59敲除的MCF7细胞聚团现象明显. TRIM59的 缺失减少了肌动球蛋白F-actin的伸缩和细胞变形迁 移, 增加了黏附分子E-cadherin表达和癌细胞附着能 力; 而TRIM59过表达则会抑制E-cadherin的表达.

研究人员通过酵母双杂交等实验, 发现TRIM59可 以通过其 RING 结构域与程序性细胞死亡因子 PDCD10相互作用. TRIM59的关键角色是以非酶活依 赖的形式竞争性结合PDCD1, 从而保护其免于被 RNFT1介导的泛素化修饰后进入p62依赖的自噬降解 途径. 这样TRIM59通过稳定PDCD10抑制了RhoAROCK信号通路、减弱细胞黏附, 进而促进乳腺癌细 胞转移. 与此一致的是, 该研究也证明了PDCD10具有 促进乳腺癌转移的功能.

在泛素化修饰研究领域, K48和K11链接的多聚泛 素化修饰通常会导致底物蛋白通过26S蛋白酶体降解, 而K63 链接的泛素化修饰一般不影响底物蛋白降解.

引用格式: 蒋德伟, 陈策实. 促癌因子TRIM59不依赖泛素化酶活性促进乳腺癌转移的机制. 中国科学: 生命科学, 2019, 49: 191-192

Jiang D W, Chen C S. TRIM59 shows an E3 ligase activity independent pro-metastasis function in breast cancer (in Chinese). Sci Sin Vitae, 2019, 49: 191-192, doi: 10.1360/N052018-00284 
该论文 ${ }^{[7]}$ 首次报道了RNFT1介导的K63链接泛素化修 饰的PDCD10被自噬相关蛋白p62识别并被溶酶体降 解. 虽然RNFT1可能是PDCD10的E3泛素连接酶，但 RNFT1 是否直接催化PDCD10泛素化、是否具有抑制 乳腺癌的功能等问题在该研究中没有深入探讨. TRIM59可以直接结合PDCD10，阻止其被RNFT1泛素 化修饰，这种非酶活依赖的功能在促进乳腺癌转移中 扮演了重要角色. 我们研究组也发现, HECT类型泛素 连接酶HECTD3 可以通过酶活非依赖功能，促进 MALT1蛋白泛素化并且保护其免受降解 ${ }^{[8]}$. 作为RING 类型泛素连接酶，TRIM59应该有自身催化的底物，其
在肿瘤中与泛素化修饰相关的功能还有待发现.

该研究 ${ }^{[7]}$ 报道了 TRIM59表达可以作为乳腺癌独 立预后因子，揭示了蛋白泛素化修饰、自噬降解调控 与肿瘤转移之间新的机制，为乳腺癌治疗提供了新的 分子靶标. 因此开发靶向破坏TRIM59与PDCD10相互 作用的小分子化合物以及活性小肽应该具有较好的临 床运用前景. 该研究还发现TRIM59在包括乳腺癌、肾 癌、肺癌、结肠癌等多种肿瘤中异常升高, 这些结果 对其他肿瘤转移机制的研究也具有重要的参考意义. 未来建立TRIM59条件性基因敲除小鼠模型，将对进一 步理解TRIM59的生理和病理功能大有帮助.

\section{参考文献}

1 Bray F, Ferlay J, Soerjomataram I, et al. Global cancer statistics 2018: GLOBOCAN estimates of incidence and mortality worldwide for 36 cancers in 185 countries. CA-A Cancer J Clinicians, 2018, 68: 394-424

2 Hanahan D, Weinberg R A. Hallmarks of cancer: the next generation. Cell, 2011, 144: 646-674

3 Gallo L H, Ko J, Donoghue D J. The importance of regulatory ubiquitination in cancer and metastasis. Cell Cycle, 2017, 16: 634-648

4 Amaravadi R, Kimmelman A C, White E. Recent insights into the function of autophagy in cancer. Genes Dev, 2016, 30: 1913-1930

5 Jia L, Zhou Z, Liang H, et al. KLF5 promotes breast cancer proliferation, migration and invasion in part by upregulating the transcription of TNFAIP2. Oncogene, 2016, 35: 2040-2051

6 Hatakeyama S. TRIM proteins and cancer. Nat Rev Cancer. 2011, 11: 792-804

7 Tan P, Ye Y, He L, et al. TRIM59 promotes breast cancer motility by suppressing p62-selective autophagic degradation of PDCD10. PLoS Biol, 2018, 16: e3000051

8 Li Y, Chen X, Wang Z, et al. The HECTD3 E3 ubiquitin ligase suppresses cisplatin-induced apoptosis via stabilizing MALT1. Neoplasia, 2013, 15: 39-IN15 